\title{
Knowledge about Diabetes and Glycemic Control among Diabetic Patients in Saudi Arabia
}

\author{
Noura A. Abouammoh ${ }^{1}{ }^{1}$ and Muteb A. Alshamrani ${ }^{2}$ \\ ${ }^{1}$ Family and Community Medicine, King Saud University, Riyadh 4545/145111, Saudi Arabia \\ ${ }^{2}$ Family and Community Medicine, Security Forces Hospital, Riyadh 3643/11481, Saudi Arabia \\ Correspondence should be addressed to Noura A. Abouammoh; nabouammoh@ksu.edu.sa
}

Received 3 October 2019; Revised 29 January 2020; Accepted 27 February 2020; Published 7 March 2020

Academic Editor: Jonathan M. Peterson

Copyright (C) 2020 Noura A. Abouammoh and Muteb A. Alshamrani. This is an open access article distributed under the Creative Commons Attribution License, which permits unrestricted use, distribution, and reproduction in any medium, provided the original work is properly cited.

\begin{abstract}
The prevalence of diabetes in various regions has attracted significant attention of the medical experts. The prevalence of diabetes is expected to increase in the future due to changes in lifestyle and unhealthy diets of individuals. The objective of the study is to identify the extent of knowledge related to diabetes and glycemic controls in various diabetic patients living in Saudi Arabia. A total of 435 patients were recruited using a random sampling technique, while following a cross-sectional design. Patients' knowledge was tested using the Michigan Diabetes Knowledge Test. Findings of the study illustrated that the problem was common among middle-aged male patients. A significant amount of knowledge related to the consumption of medicines, insulin, healthy diet, etc. was found among diabetic patients. Despite the fact that people have adequate knowledge, valuable attention is yet required to provide necessary counselling to people living in Saudi Arabia that may help them to control health risks and mortality.
\end{abstract}

\section{Introduction}

In the healthcare domain, the most proliferated disease recognized across the world is diabetes. This is evident from the increased reporting of diabetes disease which is expected to reach a figure of 366 million [1] and expected to become the seventh leading cause of death by 2030 [2]. In 2014 alone, its global prevalence was reported to be 8.5 percent [2]. The most common type of diabetes is type 2 which is observed among 90 to $95 \%$ of the diabetic population globally [3]. It has stimulated as a global health concern accounting to the highest rate of morbidity and mortality [4]. Fareed et al. [5] demonstrate that the deficiency of the insulin actions in type 2 diabetes adds to the macrovascular and microvascular complication, which mitigates the health-related quality of life (HRQoL).

The prevalence of diabetes in developing countries is relatively high. In fact, Saudi Arabia as a developing country has the highest diabetes prevalence in both regional and global landscapes [6]. The state invested about 1,142 million dollars for overcoming the progressive disease in the country which is tremendously affecting its workforce. Sabbah and Al-Shehri [7] highlighted the report of WHO stating that the country spends about $\$ 800$ for providing care of its country diabetes mellitus person, whose yearly cost reaches up to $\$ 9.6$ billion. Earlier researches have advocated that the increased prevalence of the disease has not been mitigated despite the massive investment made by the country. Fatema et al. [8] and Mohammadi [9] pinpointed that despite the advancement and development of the disease research, the patient's level of disease knowledge remains low affecting the glycemic control. It has been claimed by Marathe et al. [10] that escalated diabetes complication risk and mortality can be lowered by intensive glycemic control. Alsulaiman et al. [11] indicated that about two-thirds of Saudi diabetic patients have poor glycemic control. On the contrary, glycemic control is an essential indicator to prevent the chronic metabolic disease complications such as metabolic syndrome, cardiovascular and kidney disease, and diabetes. Among chronic metabolic diseases, glycemic control management has been a domain of interest from the past years. The glycemic index shows an increasing fasting blood glucose level after 
consuming a high carbohydrate-containing diet. The glycemic index is directly associated with different chronic metabolic diseases among the human populations [12].

Similarly, to gain a better understanding of the patient's health literacy and practices, a Malay Elderly Diabetes Self-Care Questionnaire (MEDSCaQ) was also developed by Ishak et al. [13]. The findings of the survey using this questionnaire revealed that health literacy and disease management improved with the presence of family support as well as good diabetes knowledge. The ultimate outcomes of the study include diabetes knowledge, ethnicity, family support, and comorbidities that contributed to the improvement of the disease knowledge. Likewise, paperwork by Shareef [14] stated that disease outcomes improved with pharmacist-administrated diabetes knowledge. It showed that adequate access to diabetes knowledge adds to diabetes management and a patient's control. Diabetes is a condition anticipated for rapid advancement over time. The high blood sugar can influence various cells and organs in the body if type 2 diabetes goes untreated. Side effects include eye damage, stroke, heart disease, and kidney damage. Family members disseminate genes that make them prone to possess type 2 diabetes and to be overweight [15].

Pérez-Escamilla et al. [16] examined the effect on type 2 diabetes patients' glycemic control by introducing an intervention led by the community health worker. In this regard, they used the Diabetes among Latinos Best Practices Trial (DIALBEST) and showed that lifestyle trend and ethnicity contribute to the effective prevention of the disease and improvement of glycemic control.

In a similar context, many researches have attempted to examine the factors which can contribute to the improved health of the patients stimulated by their adequate knowledge of the disease and ineffective glycemic control. The American Diabetes Association in 2015 introduced a diabetes selfmanagement education and support (DSMES) program, which studied patients' attributes and characteristics for providing them with adequate support and customized care. It includes knowledge about their convictions, ethnic practices, sociodemographic factors, and socioeconomic factors [17]. The same study also underlined that supplementing adequate knowledge of diabetes requires the provision of better support for achieving better health outcomes. Zainudin et al. [18] revealed that a gap in the disease knowledge leads to the adaptation of adverse activities affecting the patients' health outcome. Azreena et al. [19] further argued and stated that adequate health literacy is a prerequisite for effective utilization of the measures for efficient diabetes management. Sanal et al. [20] highlighted that glycemic control is substantially associated with diet and physical activity.

The increased disparity in the knowledge concerning diabetes and glycemic control contributes to the ineffective clinical control which impacts the better disease knowledge. Since optimal disease knowledge is integral for reducing the related risk factors of inadequate control, the assessment of the patient's mindfulness is critical. Therefore, the study is aimed at assessing the overall diabetes knowledge in the patients of Security Forces Hospital in Saudi Arabia. The study used a 14-item Michigan Diabetes Knowledge Test
(MDKT) questionnaire for testing the knowledge about diabetes among the patients in Security Forces Hospital, Riyadh.

\section{Material and Methods}

2.1. Participants and Statistical Sample Size Formula. The study uses a cross-sectional study design for assessing the relationship between type 2 diabetic patients' knowledge and glycemic control in Security Forces Hospital, Riyadh, Saudi Arabia. The period for the study was from January 2018 to March 2018. A total of 435 patients who visited the outpatient clinics of Security Forces Hospital, Riyadh, were recruited using a random sampling method. Random sampling in research methods denotes a variety of selection techniques in which sample participants are chosen by chance, but with an identified likelihood of selection.

2.2. Inclusion and Exclusion Criteria. The selected patients were evaluated for the inclusion criteria which involved them being diagnosed for type 2 diabetes, under care at the hospital, and above 18 years. Patients suffering from physical or mental impairment and pregnant women were not included in the study. The sample size was calculated based on the patients admitted in the outpatient clinics of Security Forces Hospital, 95\% confidence interval, and $80 \%$ power size using G-power version 3.1.9.4.

2.3. Tools and Validity and Reliability. The structured questionnaire of the 14-item MDKT was used for examining the consenting participant's diabetic knowledge. HbAlc was collected from the patients' hospital record. The knowledge of diabetes and HbA1c levels were taken as dependent variables. The independent variables involve gender, age, qualification, marital status, time since diagnosis, occupation, and present medication regime. Demographic details of patients were collected through a designed questionnaire that consists of questions related to gender, age, marital status, type of job, educational level, duration of diabetes, etc. Other information includes type of treatment provided to diabetes patients and level of education related to diabetes along with their family history of diabetes.

2.4. Questionnaire Filling Method. A self-administered 14-item MDKT questionnaire was used for gathering patients' diabetic knowledge and their basic demographic details. This instrument was used to evaluate the knowledge of the elderly in this study. The Michigan Diabetes Research and Training Center has developed the MDKT. The 14-item questionnaire is reliable and valid and thus has been majorly utilized for evaluating diabetes knowledge. The instrument is comprised of three sections. The first section collected sociodemographic data, the second section consists of the 14-item MDKT, and the third section consists of the laboratory data to be collected from the patients' records. This questionnaire originally held 23 items, though the study only included the first 14 items which address the general awareness of the diabetic disease. These items were inclusive with one right answer along with multiple wrongs. The Arabic translation of the questionnaire was obtained from the study of Azreena 
et al. [19] for sustaining context relevance, after the approval of his team. The patients selected the questionnaire language.

These items were assessed for their reliability which was found to be 0.7 and 0.71 along with adequate internal consistency (Cronbach alpha), i.e., 0.702 . Developed by the University of Michigan's Diabetes Research and Training, the MDKT was used, which is also accessible online (http:// diabetesresearch.med.umich.edu/Tools_SurveyInstruments .php\#dkt). The study was commenced after achieving authorization from the Diabetes Care Center at Riyadh Security Forces Hospital. The patients were also communicated with the study details and were included after the attainment of their written consent.

2.5. Statistical Analysis. The collected data were analyzed using IBM SPSS version 25. Categorical variables were analyzed descriptively. Frequencies and percentages were used to present continuous and categorical variables. The Kruskal-Wallis test was used to show the association between $\mathrm{HbAlc}$ levels and sociodemographic characteristics. A haemoglobin Alc (HbAlc) test measures the amount of glucose related to haemoglobin. It is the part of red blood cells that transmits oxygen from the lungs to the rest of the body. An $\mathrm{HbAlc}$ test indicates what the average amount of blood sugar connected to haemoglobin has crossed the time period of 3 months. It is a 3-month average because that is usually a red blood cell life. Different chronic conditions can be caused such as kidney disease, nerve damage, and heart disease if HbAlc levels are high.

\section{Results}

Table 1 shows the demographic details of the patients. Among 435 participants, the majority of the participants were male, i.e., $53.1 \%$, whereas the percentage of females was $46.9 \%$. The majority of the samples belong to Saudi nationality (98.6\%) and the least of the samples to nonSaudi nationality $(1.3 \%)$. Much of the sample population belongs to the age group 51-60 years (39.5\%), followed by 41-50 years $(22.1 \%)$ and $61-70$ years (16.4\%), producing a median age of 55 years. Most of the participants were married $(93.3 \%)$ as compared to single $(6.7 \%)$. The education level of the patients showed that the majority held only primary-level education (29.2\%) followed by secondarylevel education (27.1\%) and college degree (26.9\%). Family history revealed that $71.9 \%$ had a family history of glycemic control, whereas $28.1 \%$ did not. The occupation analysis revealed that $38.9 \%$ had retired, $33.5 \%$ are nonworking, and $23.9 \%$ are employed in the government sector while $23.9 \%$ worked in the private sector.

Table 2 shows the findings of the examined patient's record. The analysis showed that the majority of the patients were on medication, where $44.8 \%$ take oral medicine and $13.8 \%$ take insulin therapy alone, while $36.4 \%$ took a combination of oral and insulin therapy, whereas dietary interventions were used by $5 \%$. The diabetes family history was found for $72 \%$ of the patients, and a diagnosis age median was 11 years, where the majority reported living with diabetes for 6-15 years. Among 435 patients, the majority of the patients
Table 1: Patient demographic characteristics.

\begin{tabular}{|c|c|}
\hline Variables & Percentage \\
\hline \multicolumn{2}{|l|}{ Gender } \\
\hline Male & $53.1 \%$ \\
\hline Female & $46.9 \%$ \\
\hline \multicolumn{2}{|l|}{ Nationality } \\
\hline Saudi & $98.5 \%$ \\
\hline Non-Saudi & $1.3 \%$ \\
\hline Data unavailable & $0.2 \%$ \\
\hline \multicolumn{2}{|l|}{ Age } \\
\hline 23-30 years & $1.8 \%$ \\
\hline $31-40$ years & $10.1 \%$ \\
\hline $41-50$ years & $22.1 \%$ \\
\hline $51-60$ years & $39.5 \%$ \\
\hline $61-70$ years & $16.4 \%$ \\
\hline $71-80$ years & $10.1 \%$ \\
\hline \multicolumn{2}{|l|}{ Marital status } \\
\hline Married & $93.3 \%$ \\
\hline Single & $6.7 \%$ \\
\hline \multicolumn{2}{|l|}{ Family history } \\
\hline Present & $71.9 \%$ \\
\hline Absent & $28.1 \%$ \\
\hline \multicolumn{2}{|l|}{ Education } \\
\hline Primary level & $29.2 \%$ \\
\hline Intermediate level & $16.8 \%$ \\
\hline Secondary level & $27.1 \%$ \\
\hline College degree & $26.9 \%$ \\
\hline \multicolumn{2}{|l|}{ Employment type } \\
\hline Government sector & $23.9 \%$ \\
\hline Private sector & $3.2 \%$ \\
\hline Self-employment/business & $0.4 \%$ \\
\hline Retired & $38.9 \%$ \\
\hline Nonworking & $33.6 \%$ \\
\hline
\end{tabular}

(71.2\%) have used diabetes knowledge for disease management. The high HbAlc levels were found among the patients $(9.3 \pm 4.6 \%)$. A total of $53.3 \%$ reported poor glycemic control of levels above $8.5 \%$, while moderate glycemic control was shown by $1.8 \%$, and lastly, glycemic control below $7.0 \%$ level was observed in $14.0 \%$ patients.

The percentage of the participants who had correctly answered the questions in the MKDT questionnaire has been depicted in Table 3. It demonstrates that the difference in the normal and special diet is recognized by only $40 \%$, whereas $51.5 \%$ understood the significance of the diabetic diet in the health context. A large portion of the sample showed that they considered diabetic diet to be rich in carbohydrate, whereas $1.6 \%$ assumed that high protein should be consumed in a diabetic diet. Food high in carbohydrate and fats was correctly identified by $48 \%$ and $42 \%$ of the participants, respectively; while sugar-free food was identified by just $18.4 \%$ of the sample. Adequate awareness for the blood testing techniques was observed for $66.4 \%$ of the individuals, while only 
TABLE 2: Information from patients' records.

\begin{tabular}{lc}
\hline Variables & Percentage \\
\hline Treatment type & \\
Dietary intervention & $5.0 \%$ \\
Oral medication & $44.8 \%$ \\
Insulin therapy & $13.8 \%$ \\
Both oral and insulin therapy & $36.4 \%$ \\
Sought diabetes education & \\
Yes & $71.3 \%$ \\
No & $28.7 \%$ \\
Year since diagnosis (median years since & \\
diagnosis $=11$ years) & \\
$\quad \leq 5$ years & $26.8 \%$ \\
6-15 years & $46.0 \%$ \\
16-30 years & $24.7 \%$ \\
$>30$ years & $2.5 \%$ \\
Hb1AC levels (mean $=9.3 \pm 4.6)$ & \\
Good control: $<7.0 \%$ & $14.0 \%$ \\
Moderate control: $7.0-8.5 \%$ & $1.8 \%$ \\
Poor control: $>8.5 \%$ & $53.3 \%$ \\
\hline
\end{tabular}

TABLE 3: Awareness percentage of the participants.

\begin{tabular}{lc}
\hline MDKT items & Percentage \\
\hline N1 & 51.5 \\
N2 & 47.8 \\
N3 & 42.1 \\
N4 & 18.4 \\
N5 & 44.6 \\
N6 & 66.4 \\
N7 & 42.5 \\
N8 & 37.7 \\
N9 & 88.3 \\
N10 & 73.10 \\
N11 & 73.30 \\
N12 & 79.80 \\
N13 & 65.30 \\
N14 & 54.90 \\
\hline
\end{tabular}

$44 \%$ were able to explain $\mathrm{HbA1c}$. An adequate level of knowledge was observed among the participants for the effect of exercise $(88.3 \%)$ or infection $(73.1 \%)$ on levels of blood glucose. A total of $73.3 \%$ of the participants showed adequate levels of awareness for foot care while comorbidity awareness was found to range from 54.9 to $79.8 \%$ (Table 3).

The Kruskal-Wallis analysis was performed to understand the correlation between glycemic control as measured by $\mathrm{HbAlc}$ levels and demographic variables including age, education level, gender, and employment type. The null hypothesis for the analysis implies that there is no dependence of HbAlc levels on the variable. The null hypothesis
TABLE 4: Association between levels of HbAlc and sociodemographic variables.

\begin{tabular}{lccc}
\hline Variables & $\begin{array}{c}\text { Value of } H \\
\text { statistic }\end{array}$ & $\begin{array}{c}\text { Critical chi-squared } \\
\text { value }\end{array}$ & $P$ value \\
\hline Gender & 5.47 & 3.84 & $<0.001$ \\
Nationality & 17 & 9.5 & $>0.001$ \\
Age & 3.9 & 11.07 & $<0.001$ \\
Education level & 13.5 & 7.8 & $<0.001$ \\
Employment type & 33 & 9.48 & $<0.001$ \\
\hline
\end{tabular}

was rejected if the critical chi-squared value was less than the $H$ statistic. Based on the findings, the null hypothesis was rejected for gender, age, educational level, and employment type (Table 4).

\section{Discussion}

Healthcare practices have always been a matter of interest for various medical professionals. In the present time, a greater number of people are suffering from diabetes from a very young age. Knowledge related to diabetes can help patients to overcome the problem through healthy diet and other important safety measures. This pinpoints the idea that to which extent Saudis are aware of the knowledge related to diabetes. The findings of this study illustrate that the majority of the patients were male suffering from diabetes. The results proposed by this study contradict those provided by Fareed et al. [5]. Accordingly, the majority of the females were affected by poor glycemic controls within the region of Saudi Arabia. The reason behind the problem was the high-level consumption of unhealthy diet. It was further observed that the dietary pattern followed by the diabetic patients was unhealthy due to high-level consumption of fats, cholesterol, sodium, and free sugar.

The majority of the low level of knowledge among diabetes patients was recorded in middle age groups. Besides this, the majority of the patients had a family history of glycemic control, which thus served as one of the major causes of diabetes among patients. Alzaheb and Altemani [21] supported the idea where the majority of the patients belonging to urban areas have poor knowledge about glycemic controls. However, people with substantial background of poor glycemic controls were at greater risks of diabetes. The lack of knowledge regarding diabetes resulted in inefficient healthcare management, as patients with maximum diabetic durations were not habitual of regular exercises along with dietary controls.

Another important finding of this study indicated that the majority of diabetes patients were on medication, while few of them were recommended oral medicines. However, the diabetic population was rarely habitual in consuming a combination of insulin and oral therapy. Nguyen et al. [22] proposed slightly different results that almost half of the diabetic population was using oral medication. The study further indicated that level of HRQoL was low among patients consuming regular insulin rather than healthy dietary therapies. This pinpoints that both insulin and oral 
medicines can further create an impact on health conditions of diabetes patients. Besides this, the study indicated that the level of knowledge among various Saudi patients were seemingly high. This indicates that the level of awareness among patients served as an important determinant among diabetic patients. Sami et al. [23] indicated that poor quality of knowledge was observed among diabetic patients living in Saudi Arabia, in comparison to those living in any other states. Besides this, low level of knowledge was observed regarding the complications provided by diabetes. However, most of the people were unaware of the importance of medicines to control adverse effects of diabetes.

Other than this, knowledge related to the diabetic diet was not recorded among the majority of Saudi patients, while $50 \%$ of the patients were aware of the significance of healthy diet for controlling diabetes. For the majority of patients, a healthy diet is related to the high-level consumption of carbohydrates, whereas for others high protein must be consumed to control the effect and level of diabetes. Alramadan et al. [6] demonstrated that the majority of the people were prone to unhealthy lifestyles leading towards other healthrelated issues such as obesity. This has provided them greater difficulties in controlling their sugar and blood levels. It was further identified that the majority of patients were not consuming a healthy diet that includes daily consumption of fruits and vegetables, maximizing the risks of inadequate glycemic controls. Similarly, physical activity was also uncommon among diabetes patients. The study argued that in various countries, young people are at greater risks of poor glycemic control.

In addition to this, knowledge related to the blood testing techniques, effects of exercise, and risks associated with diabetes were found in the majority of patients, while only few of them were unable to explain the causes and effects of diabetes. Ansari et al. [24] argued that the important knowledge and safety health measures regarding poor glycemic controls were not accessible to the majority of diabetes patients in Saudi Arabia. Similarly, more than half of the diabetes patients were not following any special diet to refrain from any further health issues. Only a limited number of people were aware of the importance of control diet. This stresses the idea that still the concept related to the knowledge and healthcare measures is unclear among the majority of the patients. The results of this study demonstrated that the level of knowledge among Saudi patients was significant in comparison to results proposed by the aforementioned studies. Still, there is a need to spread valuable knowledge towards patients that are at greater risks of diabetes.

The study has reported an association of several demographic characteristics of patients with their knowledge about HbA1c levels. The higher levels of education among patients followed by the job status of the participants were strong factors for better $\mathrm{HbAlc}$ level knowledge, which was also supported by previous studies conducted in low- and middle-income countries [25-27]. Another study has asserted that patients' educational level was a substantial predictor towards their self-care practices [28]. Furthermore, improving knowledge and awareness and pharmacological intervention among diabetic patients and their family mem- bers will lead to effective health-related consequences. The findings of this study suggested that the contents of an educational intervention can be developed considering the educational status of the patients as mostly patients with low education level had poor disease knowledge. Secondly, job nature of the diabetic patients must be taken into consideration while informing diabetes education as the disease knowledge of the majority of participants was relatable to that of illiterate participants.

\section{Conclusion}

Saudi Arabia, despite high-level economic growth and development, is prone to health-related risks such as diabetes that serves as the root cause for the development of further diseases. As stated above, currently, people are at major risk of death due to diabetes and its consequences that are now highly developing among diabetes patients. The intake of regular insulin is the major prevention for further effects of diabetes. In various countries, a greater amount of money is invested in monitoring and catering to health-related issues. This indicates the need for better understanding among patients related to their healthcare.

As per the designed questionnaire, it was demonstrated that people suffering from diabetes had adequate knowledge related to the importance of healthy diet to control further effects of the disease. However, it was found that most of the patients are more towards using medicines while a limited number of patients use insulin. Other than this, healthy practices, i.e., exercise, were not common in the majority of the population, while the diet was mostly related to the greater consumption of fats. The glycemic levels were found to be low among the patients; however, limited people were aware of the important measures that are needed to be followed among diabetes patients. The study further indicated that in most of the patients, belonging to families with diabetic background was one of the major causes of its widespread among several other patients. Thus, spreading more knowledge related to diabetes and poor glycemic controls to control the transfer of the disease was recommended. It is recommended that future researchers conduct a study regarding the prevalence of the problem in various urban and rural areas of Saudi Arabia to obtain valuable knowledge.

\section{Data Availability}

The data used to support the findings of this study are available from the corresponding author upon request.

\section{Conflicts of Interest}

The authors declare that they have no conflicts of interest.

\section{Acknowledgments}

The authors are grateful to the Deanship of Scientific Research, King Saud University, for funding through the Vice Deanship of Scientific Research Chairs. We gratefully acknowledge the patients who participated in this study. 


\section{Supplementary Materials}

The first table is the questionnaire used to collect demographic data. The second table contained the 14-item MDKT questionnaire used to assess patients' knowledge on diabetes with the correct answer marked with an asterisk. (Supplementary Materials)

\section{References}

[1] O. P. Kapoor, Common Chronic Disease Patterns in Arabian Gulf, Saudi Arabia \& Yemen, 2013, http://www.bhj.org.in/ books/arabs/chap12.htm.

[2] World Health Organization, WHO mortality database. Geneva, Switzerland: World Health Organization, 2016, http://apps.who.int/healthinfo/statistics/mortality/ causeofdeath_query/.

[3] American Diabetes Association, "Diagnosis and classification of diabetes mellitus," Diabetes care, vol. 37, Supplement 1, pp. S81-S90, 2014.

[4] I. Al-Aboudi, M. Hassali, and A. Shafie, "Knowledge, attitudes, and quality of life of type 2 diabetes patients in Riyadh, Saudi Arabia," Journal of Pharmacy and Bioallied Sciences, vol. 8, no. 3, pp. 195-202, 2016.

[5] M. Fareed, N. Salam, A. T. Khoja, M. Abdulrahman, and M. Ahamed, "Knowledge of hypoglycemia and its associated risk factors among type 2 diabetes mellitus patients in diabetes Centre, Security Forces Hospital, Riyadh, Saudi Arabia," Health Sciences, vol. 6, no. 10, pp. 125-132, 2017.

[6] M. J. Alramadan, D. J. Magliano, T. H. Almigbal et al., "Glycaemic control for people with type 2 diabetes in Saudi Arabia - an urgent need for a review of management plan," $B M C$ Endocrine Disorders, vol. 18, no. 1, p. 62, 2018.

[7] K. Sabbah and A. AlShehri, "Practice and perception of self management among diabetics in Taif, KSA: Impact of demographic factors," International Journal of Medical Science and Public Health, vol. 3, no. 3, p. 277, 2014.

[8] K. Fatema, S. Hossain, K. Natasha et al., "Knowledge attitude and practice regarding diabetes mellitus among nondiabetic and diabetic study participants in Bangladesh," BMC Public Health, vol. 17, no. 1, p. 364, 2017.

[9] S. Mohammadi, "Knowledge, attitude and practices on diabetes among type 2 diabetic patients in Iran: a cross-sectional study," Science Journal of Public Health, vol. 3, no. 4, p. 520, 2015.

[10] P. H. Marathe, H. X. Gao, and K. L. Close, "American Diabetes Association standards of medical care in diabetes 2017," Journal of Diabetes, vol. 9, no. 4, pp. 320-324, 2017.

[11] T. Alsulaiman, H. Al-Ajmi, S. Al-Qahtani et al., "Control of type 2 diabetes in King Abdulaziz Housing City (Iskan) population, Saudi Arabia," Journal of Family and Community Medicine, vol. 23, no. 1, pp. 1-5, 2016.

[12] M. Imran, S. Begum, A. H. Kandhro, N. Ahmed, and R. Qasim, "The management of glycemic control in associated disorders," International Journal of Endorsing Health Science Research (IJEHSR), vol. 5, no. 2, p. 37, 2017.

[13] N. H. Ishak, S. S. Mohd Yusoff, R. A. Rahman, and A. A. Kadir, "Diabetes self-care and its associated factors among elderly diabetes in primary care," Journal of Taibah University Medical Sciences, vol. 12, no. 6, pp. 504-511, 2017.

[14] J. Shareef, "Effect of pharmacist led educational interventions on disease knowledge and glycaemic control in patients with diabetes mellitus in a University Hospital," Current Research in Diabetes \& Obesity Journal, vol. 1, no. 2, 2016.

[15] I. Mühlhauser, "Evidence-based patient education in diabetes and beyond - application to other chronic diseases," Embedding Education into Diabetes Practice, vol. 18, pp. 132-146, 2005.

[16] R. Pérez-Escamilla, G. Damio, J. Chhabra et al., "Impact of a community health workers-led structured program on blood glucose control among Latinos with type 2 diabetes: the DIALBEST trial," Diabetes Care, vol. 38, no. 2, pp. 197-205, 2014.

[17] IDF Diabetes Atlas, IDF Diabetes Atlas 5th Edition, 2011, https://www.idf.org/e-library/epidemiology-research/ diabetes-atlas/20-atlas-5th-edition.

[18] S. Zainudin, D. Ang, and A. Soh, "Knowledge of diabetes mellitus and safe practices during Ramadan fasting among Muslim patients with diabetes mellitus in Singapore," Singapore Medical Journal, vol. 58, no. 5, pp. 246-252, 2017.

[19] E. Azreena, I. Suriani, M. H. Juni, and P. Fuziah, "Factors associated with health literacy among type 2 diabetes mellitus patients attending a government health clinic," International Journal of Public Health and Clinical Sciences, vol. 3, no. 6, pp. 50-64, 2016.

[20] T. S. Sanal, N. S. Nair, and P. Adhikari, "Factors associated with poor control of type 2 diabetes mellitus: a systematic review and meta-analysis," Journal of Diabetology, vol. 3, no. 1, pp. 1-10, 2011.

[21] R. Alzaheb and A. Altemani, "The prevalence and determinants of poor glycemic control among adults with type 2 diabetes mellitus in Saudi Arabia," Diabetes, Metabolic Syndrome and Obesity: Targets and Therapy, vol. Volume 11, pp. 15-21, 2018.

[22] H. T. T. Nguyen, M. Moir, T. X. Nguyen et al., "Health-related quality of life in elderly diabetic outpatients in Vietnam," Patient Preference and Adherence, vol. Volume 12, pp. 1347-1354, 2018.

[23] W. Sami, T. Ansari, N. Shafique Butt, and M. R. B. A. Hamid, "Diabetes mellitus knowledge of type 2 diabetic patients, Almajmaah, Saudi Arabia," International Journal of Advanced Life Sciences, vol. 10, no. 4, pp. 377-385, 2017.

[24] T. Ansari, W. Sami, N. Alsubaie et al., "Assessment of knowledge, attitude, and practice of dietary pattern in patients with type 2 diabetes mellitus: a locality-based perspective study," International Journal of Medicine in Developing Countries, vol. 3, pp. 581-585, 2019.

[25] T. Kassahun, H. Gesesew, L. Mwanri, and T. Eshetie, "Diabetes related knowledge, self-care behaviours and adherence to medications among diabetic patients in Southwest Ethiopia: a cross-sectional survey," BMC Endocrine Disorders, vol. 16, no. 1, p. 28, 2016.

[26] M. K. Parakh, A. Kasi, V. Ayyappan, and S. Prabhu, "Knowledge and awareness of oral manifestations of diabetes mellitus and oral health assessment among diabetes mellitus patients- a cross sectional study," Current Diabetes Reviews, vol. 16, no. 2, pp. 156-164, 2019.

[27] A. Bukhsh, T. M. Khan, M. Sarfraz Nawaz, H. Sajjad Ahmed, K. G. Chan, and B.-H. Goh, "Association of diabetes knowledge with glycemic control and self-care practices among Pakistani people with type 2 diabetes mellitus," Diabetes, Metabolic Syndrome and Obesity: Targets and Therapy, vol. Volume 12, pp. 1409-1417, 2019.

[28] A. Bukhsh, T. M. Khan, M. Sarfraz Nawaz et al., "Association of diabetes related self-care activities with glycemic control of patients with type 2 diabetes in Pakistan," Patient Preference and Adherence, vol. Volume 12, pp. 2377-2385, 2018. 